\title{
Soziologie der Schuld
}

\author{
Volker Kruse
}

Angenommen: 22. Februar 2021 / Online publiziert: 11. Mai 2021

(C) Der/die Autor(en) 2022, korrigierte Publikation 2022

Von Haselberg, Peter: Schuldgefühle. Postnazistische Mentalitäten in der frühen Bundesrepublik. Frankfurt: Campus 2020. Herausgegeben von Michael Becker, Dirk Braunstein und Fabian Link. Eine Studie aus dem Gruppenexperiment am Institut für Sozialforschung. 252 Seiten. ISBN 978-3593-51191-7. Preis: $€ 29,95$.

Wie war es um die politische Einstellung, das politische Denken der Deutschen in den Jahren nach Ende des Zweiten Weltkriegs bestellt? Inwieweit war es noch vom Nationalsozialismus geprägt, inwieweit zeigten sich bereits demokratische Ansätze? Wie dachten die Deutschen über Konzentrationslager und die massenhafte Vernichtung des europäischen Judentums? Was konnte getan werden, um eine demokratische Einstellung und damit die Demokratie in Deutschland zu fördern?

Diese Fragen beschäftigten Max Horkheimer und Theodor W. Adorno nach ihrer Rückkehr aus ihrem Exil nach Frankfurt 1949 und mündeten in eine Reihe von Gruppenexperimenten zur politischen Mentalität der deutschen Bevölkerung. In Hessen, Bayern und Norddeutschland wurden Gruppen von acht bis sechszehn Teilnehmenden befragt. Das erhobene Datenmaterial umfasst insgesamt 121 transkribierte Gruppendiskussionen in Form von Protokollen mit 1635 Personen auf insgesamt 6392 Schreibmaschinenseiten. Diese Gruppen waren zunächst nach dem Zufallsprinzip, bald sozial homogen zusammengesetzt. Es wurde keine Repräsentativität angestrebt, wohl aber sollte ein breites soziales Spektrum erfasst werden.

Im Jahr 1955 wurde der Band „Gruppenexperiment“ veröffentlicht, herausgegeben von Horkheimer und Adorno, bearbeitet von Friedrich Pollock, der aber nur rudimentär die geleisteten Forschungsarbeiten wiedergab. Zwölf Monografien waren

\footnotetext{
V. Kruse $(\bowtie)$

Fakultät für Soziologie, Universität Bielefeld

Universitätsstraße 25, 33615 Bielefeld, Deutschland

E-Mail: volker.kruse@uni-bielefeld.de
} 
erarbeitet worden, aber ihre Publikation erschien politisch nicht opportun. Adressaten der Gruppenexperimente waren aus Sicht von Adorno und Horkheimer eher Regierungs- und hohe Verwaltungsentscheider als die breite Öffentlichkeit.

Das Institut für Sozialforschung unternimmt es nun, einen Teil der damals unveröffentlichten Monografien im Rahmen der Frankfurter Beiträge zur Soziologie und Sozialphilosophie zu publizieren. Als erste Monografie dieses Projekts ist „Schuldgefühle. Postnazistische Mentalitäten in der frühen Bundesrepublik" erschienen. Der Buchtitel stammt von den Herausgebern; der Titel im ursprünglichen Typoskript lautete: „Verhaltensweisen gegenüber dem Schuldgefühl“. Der Autor Peter von Haselberg (1908-1994) hatte vor der nationalsozialistischen Machtübernahme bei Hans Kelsen eine Dissertation über das Thema „Staatstheorie des Nationalsozialismus“ geschrieben, die jedoch aufgrund der Entlassung des Doktorvaters nicht mehr zur Promotion führte und als verschollen gilt. Haselberg ging ins argentinische Exil. 1949 kehrte er zurück und wurde Mitarbeiter am Institut für Sozialforschung.

Im Zentrum der 1950/51 durchgeführten Forschung von Haselbergs steht die Frage, wie die Deutschen mit dem Problem der kollektiven und individuellen Schuld umgingen. Als „Anreiz“ der Gruppenexperimente wurde ein Text eines amerikanischen Besatzungsoffiziers vorgespielt, der sogenannte „Colburn-Brief“, abgedruckt am Ende des Bandes. Darin äußert sich Colburn grundsätzlich positiv über die Deutschen, beklagt aber die fehlende Empathie gegenüber den nationalsozialistischen Verbrechen. Die Studie enthält zahlreiche ausgewählte Textpassagen aus den Protokollen, die schon für sich genommen eine spannende Lektüre garantieren und die von Haselberg kritisch und scharfsinnig analysiert. Dabei arbeitet er insbesondere nationalsozialistische Subtexte heraus.

Hilfreich und oft unentbehrlich zum Verständnis sind die erläuternden Fußnoten der Herausgeber zum Haupttext. Dabei werden auch sachliche Fehler in den zitierten Textpassagen korrigiert. Allerdings bleiben Alter und soziale Position der Probanden meist im Dunkeln.

Im Ergebnis lässt sich sagen, dass das Schuldproblem von den Probanden sehr wohl wahrgenommen wurde. Die nationalsozialistischen Verbrechen als solche werden in der Regel nicht grundsätzlich geleugnet. Die Diskussionsbeiträge zeigen aber die deutliche Tendenz, die kollektive Schuld des deutschen Volkes, vor allem die individuelle Schuld, wenn nicht zurückzuweisen, so doch zu relativieren und zu marginalisieren.

Die Studie von Haselbergs geht den Denk- und Sprachmustern nach, in denen der Umgang mit der eigenen kollektiven oder individuellen Schuld zum Ausdruck kommt. Dazu zählen:

- Aufrechnung der Untaten. Nationalsozialistische Verbrechen ja, aber es gab auch die zahllosen Luftangriffe auf deutsche Städte mit angeblich 250.000 Toten allein in Dresden, Vertreibungen, Kriegsverbrechen in den laufenden Kriegen in Korea und Indochina.

- Aufrechnung des Leidens. Die Juden haben gelitten, die besetzten Länder haben gelitten, aber auch das deutsche Volk hat gelitten.

- Das eigene Leid wird eher den Siegermächten zugerechnet, nicht dem NS-Regime. 
- Ungleiches Maß für gleiches Tun im Krieg: „[D]ie Anderen ... werden mit Orden und Ehrenzeichen behängt, und uns hat man dann die Leute gehängt" (S. 74).

- Es wird eine Mitschuld der Alliierten konstruiert (z. B. Versailler Vertrag). Alliierte Politiker und Diplomaten hätten nicht rechtzeitig gegen Hitler eingegriffen.

- Die Judenverfolgung erfolgte durch den Staat, die Akteure außerhalb des staatlichen Raums haben davon wenig bis nichts mitbekommen.

- Offene Empörung gegen das Regime hätte existenzielle Risiken für potenzielle Opponenten und Opponentinnen und ihre Familien bedeutet.

- Aus der Sicht der Probanden gibt es eine, wie von Haselberg formuliert, ,Weltordnung der Befehle“ (S. 112). Eine hierarchische, nach den Prinzipien Befehl und Gehorsam funktionierende Staatsordnung dispensiert die Individuen von der Verantwortung für die Mitmenschen. Das vergangene Geschehen bekommt dadurch etwas Schicksalhaftes. Die Schuldfrage stellt sich dann letztendlich nicht mehr.

Vor der eigentlichen Studie steht eine etwa 20-seitige gelungene Einleitung der Herausgeber, welche Theorie und Praxis des Gruppenexperiments vorstellt und die kognitiven und politischen Ziele herausarbeitet. Sie betonen, dass es das Anliegen dieser sich als gesellschaftskritisch verstehenden Soziologie war, nicht nur Befindlichkeiten festzustellen, sondern ein Faktor der Demokratisierung der deutschen Nachkriegsgesellschaft zu sein und die Herausbildung demokratischer Milieus zu befördern. Das Gruppenexperiment sollte nicht zuletzt die Teilnehmenden dazu anregen, die eigenen politisch-weltanschaulichen Haltungen zu überwinden.

Gegen die Vorstellung einer „Stunde Null“ bringen die Herausgeber den Begriff einer ,postnazistischen Gesellschaft“ in Stellung, ,deren politische Kultur nur in Kontinuität mit dem Nationalsozialismus voll verständlich wird“ (S. 21 f.). Dem ist grundsätzlich zuzustimmen. So sind rassistische nationalsozialistische Stereotype etwa zu „den“ Juden unübersehbar (z. B. S. 129 ff.). Andere Befunde der Studie, etwa über Konzentrationslager oder die Vernichtung der jüdischen Bevölkerung, lassen sich aber auch als ein erster Schritt zur Distanzierung vom Nationalsozialismus interpretieren. Allerdings erfolgt Kritik oft verhalten und relativierend. Schuld wird wahrgenommen, aber, so die Herausgeber treffend, ,sie kann doch nicht ins Verhältnis zur eigenen Person und zum eigenen Handeln gesetzt werden“ (S. 12).

In von Haselbergs Studie erscheint der Umgang mit kollektiver und individueller Schuld naturgemäß als spezifisch deutsches Problem der Nachkriegszeit. Heutzutage sind auch andere Kontextualisierungen denkbar, z. B. die Schuldaufarbeitung hinsichtlich der Kollaboration in besetzten Ländern während des Zweiten Weltkriegs oder der Umgang mit der eigenen kolonialistischen Vergangenheit. Man kann Peter von Haselbergs Forschung also auch als eine Fallstudie zur heiklen Frage kollektiver Schuldaufarbeitung allgemein einordnen.

Die Studie samt der Einleitung ist zudem aufschlussreich zur deutschen Soziologie der 1950er-Jahre. Die These, sie habe sich zukunftsorientiert nicht mit der nationalsozialistischen Vergangenheit beschäftigt, ist möglicherweise $\mathrm{zu}$ relativieren - wie stark, werden die weiteren Bände der Reihe zeigen. Die Frankfurter Schule der 1950er-Jahre lässt sich nicht umstandslos als empirieferne „Kulturkritik“ qualifizieren und auch nicht nur als „Theorie“, sondern hier wurde intensiv empirisch gearbeitet. Sollte sich dieser Befund in den Folgepublikationen bestätigen, fügt 
sich die Frankfurter Schule in die dezidiert empirische Ausrichtung bundesdeutscher Soziologie dieser Zeit ein.

Funding Open Access funding enabled and organized by Projekt DEAL.

Open Access Dieser Artikel wird unter der Creative Commons Namensnennung 4.0 International Lizenz veröffentlicht, welche die Nutzung, Vervielfältigung, Bearbeitung, Verbreitung und Wiedergabe in jeglichem Medium und Format erlaubt, sofern Sie den/die ursprünglichen Autor(en) und die Quelle ordnungsgemäß nennen, einen Link zur Creative Commons Lizenz beifügen und angeben, ob Änderungen vorgenommen wurden.

Die in diesem Artikel enthaltenen Bilder und sonstiges Drittmaterial unterliegen ebenfalls der genannten Creative Commons Lizenz, sofern sich aus der Abbildungslegende nichts anderes ergibt. Sofern das betreffende Material nicht unter der genannten Creative Commons Lizenz steht und die betreffende Handlung nicht nach gesetzlichen Vorschriften erlaubt ist, ist für die oben aufgeführten Weiterverwendungen des Materials die Einwilligung des jeweiligen Rechteinhabers einzuholen.

Weitere Details zur Lizenz entnehmen Sie bitte der Lizenzinformation auf http://creativecommons.org/ licenses/by/4.0/deed.de.

Volker Kruse Prof. Dr., emeritierter Professor der Universität Bielefeld. Forschungsschwerpunkte: Geschichte der Soziologie, Historische Soziologie, Kriegsgesellschaftstheorie. Aktuelle Publikationen: „Hochschulexperimentierplatz Bielefeld“. 50 Jahre Fakultät für Soziologie (hrsg. mit T. Strulik), Bielefeld 2019. 